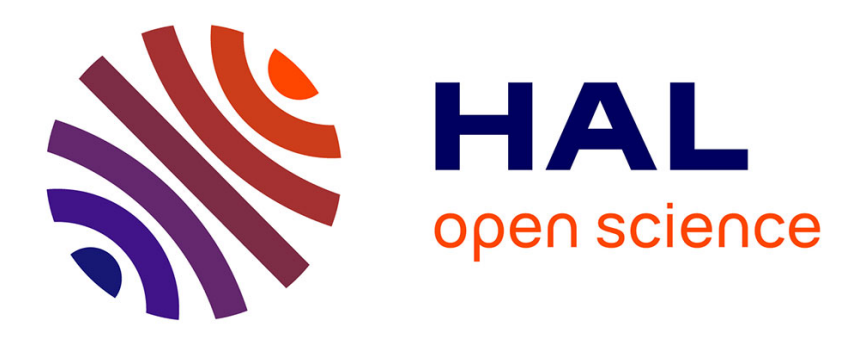

\title{
The localization transition on the Bethe lattice
}

H. Kunz, B. Souillard

\section{- To cite this version:}

H. Kunz, B. Souillard. The localization transition on the Bethe lattice. Journal de Physique Lettres, 1983, 44 (11), pp.411-414. 10.1051/jphyslet:019830044011041100 . jpa-00232210

\section{HAL Id: jpa-00232210 https://hal.science/jpa-00232210}

Submitted on 1 Jan 1983

HAL is a multi-disciplinary open access archive for the deposit and dissemination of scientific research documents, whether they are published or not. The documents may come from teaching and research institutions in France or abroad, or from public or private research centers.
L'archive ouverte pluridisciplinaire HAL, est destinée au dépôt et à la diffusion de documents scientifiques de niveau recherche, publiés ou non, émanant des établissements d'enseignement et de recherche français ou étrangers, des laboratoires publics ou privés. 


\title{
LE JOURNAL DE PHYSIQUE-LETTRES
}

J. Physique - LETTRES 44 (1983) L-411 - L-414

1er JUIN 1983, PAGE L-411

Classification

Physics Abstracts

$71.30-71.50$

\section{The localization transition on the Bethe lattice}

\author{
H. Kunz (*) \\ Institut de Physique Théorique, EPFL, Lausanne, \\ CH-1006 Lausanne, Switzerland \\ and B. Souillard (**) \\ Centre de Physique Théorique, Ecole Polytechnique, \\ F-91128 Palaiseau, France
}

(Reçu le 24 mars 1983, accepté le 14 avril 1983)

\begin{abstract}
Résumé. - Nous présentons des résultats exacts concernant la transition de localisation sur le réseau de Bethe. Sous certaines hypothèses, nous prouvons l'existence d'une transition entre le régime localisé et le régime étendu quand on varie l'énergie ou le désordre. La densité d'états est analytique aux seuils de mobilité et l'exposant $v$ gouvernant la divergence de la longueur de localisation est trouvé égal à 1 .

Abstract. - We present exact results concerning the localization transition on the Bethe lattice. In a certain number of situations, we prove the existence of a transition from extended to localized regime when varying the energy or the disorder. The density of states is analytic at the mobility edges and the exponent $v$ governing the divergence of the localization length is found equal to 1.
\end{abstract}

The question of the nature of the states of an electron in a random potential, the so-called localization problem receives much attention presently. The construction however of an approximate theory describing both localized and extended states proved to be difficult, although recently Götze [1] found one describing both the localized and the extended regimes. Another difficulty with this problem lies in the fact that no exactly soluble models are known, in contrast to phase transition problems, where soluble models have proven to be very helpful. The simplest model of the mean-field type, one could think of, is a Hamiltonian on the complete lattice with random offdiagonal elements (the Wigner model of energy levels); this model however possesses only extend-

(*) Supported by the Fonds National Suisse de la Recherche Scientifique.

$\left({ }^{* *}\right)$ Groupe de recherche 048 du CNRS. 
ed states, and does not throw light on the localization transition [5]. The next simplest model is the Anderson model on a Bethe lattice, i.e. a tight-binding model with diagonal disorder on this particular graph. It should present both localized and extended states and therefore a mobility edge. As a matter of fact this model was first studied by Abou-Chacra, Anderson, Thouless [2, 3] in 1973. These authors showed that a self-consistent approximation developed for the study of localization becomes exact on this lattice. Furthermore, they showed the existence in the energydisorder phase-diagram of a domain of stability of localized states, providing hence an estimate on the possible mobility edges. A Monte-Carlo simulation of the problem confirmed the results obtained analytically for the stability limit, and gave some information on the behaviour of the system in the non localized regime. They did not however show that truly extended states exist in the region where localized states are not stable, nor did they analyse the neighbourhood of the mobility edge. It would be of clear theoretical interest to solve this model completely in order to test approximate theories; furthermore, if this model is really the analogue of a mean-field model for the localization problem this would allow to compute the "classical exponents " for this transition.

We have further analysed this model with mathematical rigor, and have obtained a certain number of new results. First of all, we obtain the existence of mobility edges by rigorously proving the existence of localized and extended states in appropriate regions of energy or disorder. This is the first model for which the Anderson-Mott transition is proved. Moreover, we show that the density of states is analytic at the mobility edge and that the localization length diverges with an exponent $v=1$.

Let us describe more precisely our results. The Bethe lattice is an infinite graph $\mathrm{G}$, with no closed loops and a fixed coordination number $K+1$ at each vertex. The Hamiltonian $H$ that we study is defined by

$$
(H \Psi)(x)=-\sum_{y \in N(x)} \Psi(y)+w(x) \Psi(x),
$$

where the sum runs over all points which are nearest neighbours of the point $x$, on $\mathrm{G}$. The « potential " $\{w(x)\}_{x \in \mathbf{G}}$ is given as a set of independent random variables with a common probability distribution of density $\frac{1}{W} r\left(\frac{w}{W}\right)$; large $W$ corresponds to strong disorder and small $W$ to a weak one. Finally, let us consider our Hamiltonian restricted to a box $\Lambda$, which will increase to $\mathrm{G}$ in the thermodynamic limit. We will denote by $e_{\alpha}$ and $\Psi_{\alpha}$ its eigenvalues and corresponding'normalized eigenvectors.

We can introduce a correlation function at energy $e$ defined by

$$
\bar{\rho}(x ; e)=\lim _{\Lambda \uparrow \mathrm{G}}\left\langle\sum_{\alpha} \delta\left(e-e_{\alpha}\right)\left|\Psi_{\alpha}(0) \Psi_{\alpha}(x)\right|\right\rangle_{\Lambda},
$$

where the brackets mean averaging over the disorder.

On the Bethe lattice we show that this function decays always exponentially, but we prove that at strong disorder $\sum_{x:|x|=r} \bar{\rho}(x ; e)$ decays exponentially as a function of $r$, for all energies $e$. This implies, according to a general result we proved earlier [4], that, for almost all potentials, the spectrum is pure point only, and the static conductivity, as given by the Green-Kubo formula vanishes. This corresponds to complete localization of all states, at strong disorder. The same results also hold at weak disorder, but for energies lying at the edges of the spectrum, at least if the density $\frac{1}{W} r\left(\frac{w}{W}\right)$ of the probability distribution of the potential has an unbounded support, e.g. is a Gaussian or a Cauchy distribution. In the middle of the spectrum, i.e. for small energies, $\sum_{x} \bar{\rho}(x ; e)=\infty$, even for a Cauchy distribution, at weak disorder. 
One could define mobility edges, therefore, as those energies for which $\sum_{x} \bar{\rho}(x ; e)$ goes from a finite value to an infinite one; rather surprisingly, on the Bethe lattice, this definition coincides with the limit of stability for localized states obtained in reference [2].

In order to discuss the extended states, we study the function $\left\langle\left|G_{e+i \varepsilon}(0, x)\right|^{2}\right\rangle$, where $G_{e+i \varepsilon}(0, x)$ denotes the Green's function at complex energy $e+i \varepsilon$. We prove that at weak disorder, such quantities remain bounded, when $\varepsilon$ goes to zero, and this uniformly in $e$, when the energy $e$ is near the middle of the band; this implies that in this domain, for almost all potentials, the spectrum of $H$ is absolutely continuous only. In physical terms, this means that the states are not square integrable and that a particle, initially localized in some finite region, will go away to infinity. Our proof of this fact requires the finiteness of the second moment of the density $r(w)$; we cannot conclude therefore that we have extended states in the middle of the spectrum for a Cauchy distribution, although we know that $\sum_{x} \bar{\rho}(x ; e)$ diverges in this case. In fact, quite generally, we cannot exclude the possibility that in some intermediate range of energies, the states are extended or localized in an unusual way, or even that localized and extended states coexist.

These results provide the first mathematical proof of Anderson transition in any model.

We want however to go further and study the mobility edges.

In order to do so, we need however to make more specific assumptions on the density $r(w)$. For technical reasons, it has to be " close " in some sense to a Cauchy distribution, but can have all its moments finite; the results however can be shown to hold for a much broader class of distribution if some reasonable additional assumption is made. First of all, we prove that the density of states is an analytic function of the energy, even at the mobility edge. Secondly, we prove that the localization length $\xi$, defined by

$$
\xi(e)=\frac{\sum_{x}|x| \bar{\rho}(x ; e)}{\sum_{x} \bar{\rho}(x ; e)},
$$

diverges as $\xi(e) \sim\left(e-e_{\mathrm{c}}\right)^{-1}$, yielding the value 1 for the critical exponent $v$.

Let us give some hints about the proofs.

Using the general approach developed in [4], we obtain for the density of states $D(e)$, the expression

$$
D(e)=\int_{-\infty}^{+\infty} \mathrm{d} y v_{e}(y) v_{e}\left(y^{-1}\right)
$$

where $v_{e}(y)$ is a probability density solution of the non linear integral equation

$$
v_{e}(y)=\frac{1}{W} \int r\left(\frac{e+y+\sum_{j=1}^{K} y_{j}^{-1}}{W}\right) \prod_{j=1}^{K} v_{e}\left(y_{j}\right) \mathrm{d} y_{j} .
$$

The correlation functions $\bar{\rho}(x ; e)$, can be expressed as

$$
\bar{\rho}(x ; e)=\int|y|^{-1} v_{e}\left(y^{-1}\right)\left(T^{|x|} v_{e}\right)(y) \mathrm{d} y,
$$

where $T$ is a linear integral operator of kernel

$$
T\left(y^{\prime} \mid y\right)=|y|^{-1} \frac{1}{W} \int r\left(\frac{e+y^{\prime}+y^{-1}+\sum_{j=2}^{K} y_{j}^{-1}}{W}\right) \prod_{j=2}^{K} v_{e}\left(y_{j}\right) \mathrm{d} y_{j} .
$$


Using Leray-Schauder degree theory, we prove that the non-linear equation (5) has a unique positive solution $v_{e}(y)$, which can be shown to be analytic in $e$. From this follows the analyticity of the density of states in energy. The study of $\bar{\rho}(x ; e)$ requires an analysis of the operator $T$. It can be proven that this operator has discrete spectrum and a maximal non degenerate positive eigenvalue $\lambda(e)$, strictly less than one.

The asymptotic behaviour of $\bar{\rho}(x ; e)$ will then be governed by $\lambda^{|x|}(e)$. Since there are $K^{|x|}$ points at distance $|x|$ of the origin, we see that $\sum_{x} \bar{\rho}(x ; e)$ will be finite if $K \lambda(e)<1$. This condition can be proven to be satisfied at strong disorder, but violated at weak disorder near the middle of the band. The mobility edge $e_{\mathrm{c}}$ should be given by $K \lambda\left(e_{\mathrm{c}}\right)=1$. This corresponds also to the limit of stability of localized states of reference [2]. Since $\lambda(e)$ can be shown to be analytic in $e$, it follows that the localization length diverges like $\left(e-e_{\mathrm{c}}\right)^{-1}$.

In order to study the absolutely continuous spectrum, we start from the following expression for $\left\langle\left|G_{e+i \varepsilon}(0,0)\right|^{2}\right\rangle$

$$
\left\langle\left|G_{e+i \varepsilon}(0,0)\right|^{2}\right\rangle=\int_{0}^{\infty} \int_{0}^{\infty} y_{1} y_{2} \mathrm{~d} y_{1} \mathrm{~d} y_{2} g_{\varepsilon}^{K+1}\left(y_{1}, y_{2}\right) W_{\varepsilon}\left(y_{1}, y_{2}\right),
$$

where

$$
W_{\varepsilon}\left(y_{1}, y_{2}\right)=\hat{r}\left[\frac{W\left(y_{1}^{2}-y_{2}^{2}\right)}{2}\right] \exp -\frac{\varepsilon-i e}{2} y_{1}^{2}-\frac{\varepsilon+i e}{2} y_{2}^{2}
$$

and $g_{\varepsilon}$ is solution of the integral equation

$$
g_{\varepsilon}\left(y_{1}, y_{2}\right)=\int_{0}^{\infty} \int_{0}^{\infty} \mathrm{d} r \mathrm{~d} s \mathfrak{J}_{0}\left(y_{1} r\right) \mathfrak{J}_{0}\left(y_{2} s\right) \frac{\partial^{2}}{\partial r \partial s} W_{\varepsilon}(r, s) g_{\varepsilon}^{K}(r, s),
$$

$\mathfrak{J}_{0}(x)$ being the usual Bessel function.

Extended states are associated to solutions of equation (10), decaying fast enough in $y_{1}$ and $y_{2}$ when $\varepsilon=0$.

The solution is constructed at weak disorder and small energies, by some iterative procedure (Newton method), starting from a Gaussian which is a solution at zero disorder. The applicability of this approach is guaranteed only by good properties of the derivative of the non-linear operator defined by (10), at the Gaussian solution. We control this derivative by computing explicitly the spectrum of the corresponding linear operator in some appropriate functional space.

Detailed proofs of all these results will be published elsewhere.

We note also that in order to compare the Bethe lattice results with d-dimensional results, one has to reinterpret the exponent $v=1$ obtained here as $v=1 / 2$ and this has some consequences on the upper critical dimension and on the exponents of the localization problem which will be mentioned in another note.

\section{References}

[1] GöTZE, « The conductor-Non Conductor Transition in strongly disordered three-dimensional systems " : Recent Dev. Cond. Mat. Phys. 1 (Plenum Press, New York) 1981, p. 133.

[2] Abou-Chacra, R., Anderson, P. W., Thouless, D. J., J. Phys. C 6 (1973) 1734.

[3] Abou-Chacra, R., Thouless, D. J., J. Phys. C 7 (1974) 65.

[4] Kunz, H., Souillard, B., Commun. Math. Phys. 78 (1980) 201.

[5] Wigner, E. P., Ann. Math. 62 (1955) 548 ; 67 (1958) 325. That this model exhibits d.c. conductivity was shown in Wegner, S. J., Phys. Rev. B 19 (1979) 783. 